\title{
Competitive stochastic noises in coherently driven two-level atoms and quantum interference
}

\author{
P. Adam $\dagger, \ddagger$, A. Karpati $\dagger, \ddagger$, W. Gawlik $\S$, and J. Janszky $\dagger, \ddagger$ \\ $\dagger$ Department of Nonlinear and Quantum Optics, Research Institute for Solid State \\ Physics and Optics, Hungarian Academy of Sciences, \\ P.O. Box 49, H-1525 Budapest, Hungary \\ ¥Institute of Physics, University of Pécs, \\ Ifjúság út 6. H-7624 Pécs, Hungary \\ $\S$ Marian Smoluchowski Physical Institute, \\ Jagellonian University, Reymonta 4, 30-059 Kraków, Poland
}

\begin{abstract}
A system of coherently-driven two-level atoms is analyzed in presence of two independent stochastic perturbations: one due to collisions and a second one due to phase fluctuations of the driving field. The behaviour of the quantum interference induced by the collisional noise is considered in detail. The quantum-trajectory method is utilized to reveal the phase correlations between the dressed states involved in the interfering transition channels. It is shown that the quantum interference induced by the collisional noise is remarkably robust against phase noise. This effect is due to the fact that the phase noise, similarly to collisions, stabilizes the phase-difference between the dressed states.
\end{abstract}

PACS numbers: 42.50.Lc, 42.50.Gy

Submitted to: J. Opt. B: Quantum Semiclass. Opt. 


\section{Introduction}

Quantum interference is one of the most intriguing phenomena of quantum mechanics. Over the past decade several effects in atom-light interaction have been predicted and demonstrated experimentally, which have their origin in quantum interference [1, 2]. Some characteristic examples are reduction and cancellation of absorption [3, 4, 5, 6. 7, 8, 9] and spontaneous emission [10, 11, 12, 13, and narrow resonances in fluorescence 14, 15, 16.

A prerequisite of quantum interference between the transition channels is the existence of some stable time correlation of the atomic system under consideration. This time correlation is usually achieved by coherent coupling of atomic levels in multilevel systems. Noises on the other hand usually destroy all correlations in the system. Under special circumstances, however, even the incoherent perturbation can be responsible for the quantum interference [17, 18, 19. In a recent paper [20, a system of twolevel atoms strongly driven by a coherent light field and perturbed by collisional noise was analyzed by the quantum-trajectory method [21, 22, 23, 24, 25, 26. It was shown that collisional noise can unexpectedly create phase correlation between the neighboring atomic dressed states. This phase correlation is responsible for quantum interference between dressed state transition channels, resulting in anomalous modifications of the resonance fluorescence spectra found experimentally earlier [27.

Another typical example of a stochastic perturbation of a system of coherently driven two-level atoms is the phase noise of the laser field. The effect of the linewidth of the driving laser on the spectrum has been widely investigated in the literature [29, 30, 31, 32, 33, but no quantum interference effects have been found. In reference [34, however, it is suggested that an interference phenomenon similar to that induced by collisional noise can appear due to a large phase noise of the driving laser field. The consideration presented there is valid only in the case when the correlation between the atomic density matrix and the phase noise can be neglected. The assumption is rather unphysical and it is not valid, for instance, for Gaussian noise.

In this paper we present the detailed analysis of a system of coherently driven two level atoms, when both collisional and phase noises are present. We carry out the complete quantum-trajectory simulation of the system, which reveals the underlying physical processes in quantum interference phenomena.

The paper is organized as follows. In section 2 the model describing the system is introduced, and the master equation is constructed for the atomic density operator as a set of ordinary differential equations. In section 3 the resonance fluorescence spectrum is obtained. In section 4 the quantum-trajectory analysis of the problem is carried

out. Based on this, quantum interference effects are discussed in the case when both collisional and phase noises are present. Section 5 summarizes our results. 


\section{The model}

Let us consider a system of two-level atoms driven by a coherent laser field, incoherently perturbed by elastic, dephasing collisions of the atoms. Furthermore let us assume, that the driving laser field has a finite bandwidth due to its phase noise. We use the following notation: $\omega_{a}(t)$ is the fluctuating atomic transition frequency describing the stochastic perturbation due to collisions, $\varphi(t)$ is the randomly varying laser phase, $\omega_{L}$ is the frequency of the laser, $\Omega$ is the Rabi frequency, while $S^{z}, S^{+}, S^{-}$are the atomic operators defined on the ground state $(|g\rangle)$ - excited state $(|e\rangle)$ basis:

$$
S^{z}=\left(\begin{array}{cc}
1 / 2 & 0 \\
0 & -1 / 2
\end{array}\right), S^{+}=\left(\begin{array}{ll}
0 & 1 \\
0 & 0
\end{array}\right), S^{-}=\left(\begin{array}{cc}
0 & 0 \\
1 & 0
\end{array}\right) .
$$

The functions $\omega_{a}(t)$ and $\varphi(t)$ are supposed to describe Gaussian noise:

$$
\begin{aligned}
& \omega_{a}(t)=\omega_{a}+\delta \omega_{a}(t), \\
& \left\langle\delta \omega_{a}(t) \delta \omega_{a}\left(t^{\prime}\right)\right\rangle=2 \Gamma \delta\left(t-t^{\prime}\right), \\
& \frac{\mathrm{d}}{\mathrm{dt}} \varphi(t)=\vartheta(t), \\
& \left\langle\vartheta(t) \vartheta\left(t^{\prime}\right)\right\rangle=2 L \delta\left(t-t^{\prime}\right),
\end{aligned}
$$

where $\Gamma$ and $L$ stand for the magnitude of the corresponding stochastic noises. The phase noise and the noise in the atomic resonance frequency are independent, $\left\langle\delta \omega_{a}(t) \vartheta\left(t^{\prime}\right)\right\rangle=0$. Under such circumstances, the atomic Hamiltonian, in the interaction picture and rotating wave approximation, is of the form

$$
H=\hbar\left(\omega_{a}(t)-\omega_{L}\right) S^{z}+\frac{1}{2} \hbar \Omega\left(e^{-i \varphi(t)} S^{-}+S^{+} e^{i \varphi(t)}\right) .
$$

We remark, that the interaction picture is defined via the noiseless interaction-free Hamiltonian

$$
H_{0}=\hbar \omega_{a}^{(g)}|g\rangle\left\langle g\left|+\hbar \omega_{a}^{(e)}\right| e\right\rangle\langle e|,
$$

where $\omega_{a}^{(g)}$ and $\omega_{a}^{(e)}$ are the original (thus the mean value) frequencies of the ground state and excited state of the atom. The noise due to collision is taken into account in the interaction part of the Hamiltonian.

The master equation describing the time evolution of the system is of the form

$$
\dot{\rho}=\frac{1}{i \hbar}[H, \rho]+(\mathcal{L} \rho)_{\mathrm{sp}},
$$

where the linear term

$$
(\mathcal{L} \rho)_{\mathrm{sp}}=\gamma\left(-\frac{1}{2}\left(S^{+} S^{-} \rho+\rho S^{+} S^{-}\right)+S^{-} \rho S^{+}\right)
$$

describes the spontaneous emission and $\gamma$ is the natural linewidth. 
The master equation (8) is equivalent to the following stochastical differential equations for the matrix elements of the density operator, written on the ground state $(|g\rangle)$ - excited state $(|e\rangle)$ basis:

$$
\begin{aligned}
\dot{\rho}_{e e}= & \frac{1}{2} i \Omega\left(e^{i \varphi(t)} \rho_{g e}-e^{-i \varphi(t)} \rho_{e g}\right)-2 \gamma \rho_{e e}, \\
\dot{\rho}_{e g}= & i(\Delta+\delta \omega(t)) \rho_{e g}-\frac{1}{2} i \Omega e^{i \varphi(t)}\left(\rho_{e e}-\rho_{g g}\right) \\
& -\gamma \rho_{e g}, \\
\dot{\rho}_{g e}= & -i(\Delta+\delta \omega(t)) \rho_{g e}+\frac{1}{2} i \Omega e^{-i \varphi(t)}\left(\rho_{e e}-\rho_{g g}\right) \\
& -\gamma \rho_{g e}, \\
\dot{\rho}_{g g}= & -\frac{1}{2} i \Omega\left(e^{i \varphi(t)} \rho_{g e}-e^{-i \varphi(t)} \rho_{e g}\right)+2 \gamma \rho_{e e},
\end{aligned}
$$

where $\Delta=\omega_{a}-\omega_{L}$ is the detuning of the laser from the atomic resonance frequency. The stochastic differential equations can be reduced to ordinary differential equations by using the theory of multiplicative stochastic processes [35]. We summarize the main steps of this method briefly. The first step is to introduce new variables in the form $\chi_{1}=\rho_{e g}, \chi_{2}=\rho_{g e} e^{2 i \varphi}, \chi_{3}=\rho_{g g} e^{i \varphi}, \chi_{4}=\rho_{e e} e^{i \varphi}$. The differential equation system in these new variables is linear and does not contain the variable $\varphi(t)$ explicitly, only its time-derivative $\vartheta(t)$ :

$$
\dot{\chi}_{i}=\sum_{j} M_{i j} \chi_{j}+F_{i j}(t) \chi_{j}
$$

where

$$
\begin{gathered}
M=\left[\begin{array}{cccc}
i \Delta-\gamma & 0 & \frac{1}{2} i \Omega & -\frac{1}{2} i \Omega \\
0 & -i \Delta-\gamma & -\frac{1}{2} i \Omega & \frac{1}{2} i \Omega \\
\frac{1}{2} i \Omega & -\frac{1}{2} i \Omega & 0 & 2 \gamma \\
-\frac{1}{2} i \Omega & \frac{1}{2} i \Omega & 0 & -2 \gamma
\end{array}\right] \\
F(t)=\left[\begin{array}{cccc}
i \delta \omega_{a}(t) & 0 & 0 & 0 \\
0 & 2 i \vartheta(t)-i \delta \omega_{a}(t) & 0 & 0 \\
0 & 0 & i \vartheta(t) & 0 \\
0 & 0 & 0 & i \vartheta(t)
\end{array}\right] .
\end{gathered}
$$

According to the theory of multiplicative stochastic processes the equation for the ensemble averages can be written as

$$
\frac{\mathrm{d}}{\mathrm{dt}}\left\langle\chi_{i}\right\rangle=\sum_{j}\left(M_{i j}+\sum_{k} Q_{i k k j}\right)\left\langle\chi_{j}\right\rangle,
$$

where $Q_{i j k l}$ is defined by $\left\langle F_{i j}(t) F_{k l}\left(t^{\prime}\right)\right\rangle=2 Q_{i j k l} \delta\left(t-t^{\prime}\right)$. The quantity $Q_{i j k l}$ has the following form in our case:

$$
Q_{i j k l}=-\delta_{i j} \delta_{k l}\left[\begin{array}{cccc}
\Gamma & -\Gamma & 0 & 0 \\
-\Gamma & 4 L+\Gamma & L & L \\
0 & 2 L & L & L \\
0 & 2 L & L & L
\end{array}\right]_{i k}
$$


leading to

$$
\frac{\mathrm{d}}{\mathrm{dt}}\left\langle\chi_{i}\right\rangle=\sum_{j} N_{i j}\left\langle\chi_{j}\right\rangle
$$

where

$$
N=\left[\begin{array}{cccc}
i \Delta-\Gamma-\gamma & 0 & \frac{1}{2} i \Omega & -\frac{1}{2} i \Omega \\
0 & -i \Delta-\gamma-4 L-\Gamma & -\frac{1}{2} i \Omega & \frac{1}{2} i \Omega \\
\frac{1}{2} i \Omega & -\frac{1}{2} i \Omega & -L & 2 \gamma \\
-\frac{1}{2} i \Omega & \frac{1}{2} i \Omega & 0 & -L-2 \gamma
\end{array}\right]
$$

Equations for the ensemble averages of $\chi_{1}^{\prime}=\rho_{e g} e^{-i \varphi(t)}, \chi_{2}^{\prime}=\rho_{g e} e^{i \varphi(t)}, \chi_{3}^{\prime}=\rho_{g g}, \chi_{4}^{\prime}=\rho_{e e}$ and $\chi_{1}^{\prime \prime}=\rho_{e g} e^{-2 i \varphi(t)}, \chi_{2}^{\prime \prime}=\rho_{g e}, \chi_{3}^{\prime \prime}=\rho_{g g} e^{-i \varphi(t)}, \chi_{4}^{\prime \prime}=\rho_{e e} e^{-i \varphi(t)}$ can be obtained similarly, resulting in

$$
\begin{aligned}
& \frac{\mathrm{d}}{\mathrm{dt}}\left\langle\chi_{i}^{\prime}\right\rangle=\sum_{j} N_{i j}^{\prime}\left\langle\chi_{j}^{\prime}\right\rangle \\
& \frac{\mathrm{d}}{\mathrm{dt}}\left\langle\chi_{i}^{\prime \prime}\right\rangle=\sum_{j} N_{i j}^{\prime \prime}\left\langle\chi_{j}^{\prime \prime}\right\rangle,
\end{aligned}
$$

where

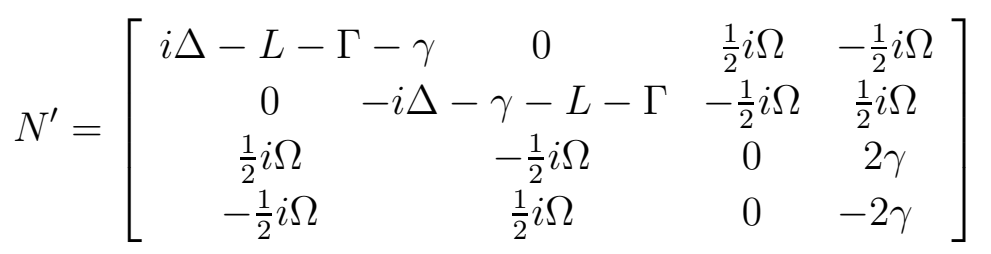

and

$$
N^{\prime \prime}=\left[\begin{array}{cccc}
i \Delta-4 L-\Gamma-\gamma & 0 & \frac{1}{2} i \Omega & -\frac{1}{2} i \Omega \\
0 & -i \Delta-\gamma-\Gamma & -\frac{1}{2} i \Omega & \frac{1}{2} i \Omega \\
\frac{1}{2} i \Omega & -\frac{1}{2} i \Omega & -L & 2 \gamma \\
-\frac{1}{2} i \Omega & \frac{1}{2} i \Omega & 0 & -L-2 \gamma
\end{array}\right] .
$$

The linear differential equation system composed from equations (19), (21), and (22) completely describes the time evolution of the ensemble averages $\left\langle\rho_{g g}\right\rangle,\left\langle\rho_{e g}\right\rangle,\left\langle\rho_{g e}\right\rangle$, and $\left\langle\rho_{e e}\right\rangle$.

\section{The resonance fluorescence spectrum}

In the dipole approximation, the resonance fluorescence spectrum $S(\omega)$ can be calculated as the real part of the two-time correlation function defined as

$$
\Gamma_{1}^{N}(\omega)=\lim _{t \rightarrow \infty} \int_{0}^{\infty} \exp (-i \omega \tau)\left\langle\left\langle S^{+}(t+\tau) S^{-}(t)\right\rangle\right\rangle d \tau .
$$

Double brackets denote quantum mechanical and stochastical averaging. The two-time average $\left\langle\left\langle S^{+}(t+\tau) S^{-}(t)\right\rangle\right\rangle$ can be expressed by one-time averages using the quantum regression theorem:

$$
\left\langle\left\langle S^{+}(t+\tau) S^{-}(t)\right\rangle\right\rangle=\left[e^{N^{\prime \prime} \tau}\right]_{22}\left\langle\chi_{1}^{\prime}(t)\right\rangle+\left[e^{N^{\prime \prime} \tau}\right]_{24}\left\langle\chi_{4}^{\prime}(t)\right\rangle
$$


After some calculation one can obtain the correlation function:

$$
\Gamma_{1}^{N}(\omega)=\frac{\Omega^{2}}{4 \gamma \Omega^{\prime}} \frac{p_{1}(\omega) p_{2}(\omega)-\gamma f(\omega) p_{2}(\omega)+\frac{1}{2} \Omega^{2}}{p_{1}(\omega) p_{2}(\omega) p_{3}(\omega)+\Omega^{2} p_{4}(\omega)},
$$

where

$$
\begin{aligned}
\Omega^{\prime} & =\frac{\Delta^{2}}{\Gamma+L+\gamma}+\Gamma+L+\gamma+\frac{\Omega^{2}}{2 \gamma}, \\
p_{1}(\omega) & =i \omega+L+2 \gamma \\
p_{2}(\omega) & =i \omega-i \Delta+4 L+\Gamma+\gamma, \\
p_{3}(\omega) & =i \omega+i \Delta+\gamma+\Gamma, \\
p_{4}(\omega) & =i \omega+2 L+\Gamma+\gamma, \\
f(\omega) & =\left(1+\frac{i \Delta}{\Gamma+L+\gamma}\right)\left(1-\frac{2 \gamma}{i \omega+L}\right) .
\end{aligned}
$$

Equation (27), in certain limits, coincides with the spectra described hitherto. Specifically, when there is no detuning, the following cases can be distinguished. In the absence of both phase noise and collisions (when $L \ll \Omega, \Gamma \ll \Omega$ ), it yields the familiar Mollow-triplet [36]. In the case of increasing phase noise and the absence of collisions, the spectrum will be of a Gaussian shape in the high noise limit [31. Increasing collision rate in the absence of phase noise, the Mollow triplet turns into a doublet structure. In the high collision rate limit one obtains a spectrum with a narrow dip at zero frequency that has its origin in quantum interference [20, 27].

The main advantage of the description presented here is that it enables us to investigate the limit of high collision rate and high phase noise simultaneously. In the case of large phase noise, there is no dip in the spectrum. Therefore one expects that phase noise demolishes the quantum interference induced by collisions which is, according to reference [20], the reason behind the appearance of the dip. Our intention is to examine how robust this interference, introduced in fact by a separate noise process, is against the competing phase noise.

The resonance fluorescence spectrum in the case of the high collision rate case is shown in figure 1 at different phase noise parameters, illustrating the disappearance of the dip.

All parameters given in the figures are accessible experimentally: $\Gamma$ can be easily adjusted by appropriate buffer gas pressure, while $L$ can be also be tailored by manipulating the laser cavity, such as, e.g. in [28].

It can be noticed, that the dip disappears at $L / \Omega \approx 2$, a relatively intense phase noise. This suggests, that the quantum interference is indeed robust against phase noise, which will be explained in what follows.

\section{Quantum-trajectory analysis of the interference}

The stochastically induced quantum interference in coherently driven two-level system can be analyzed with the aid of quantum-trajectory method. This method is capable 


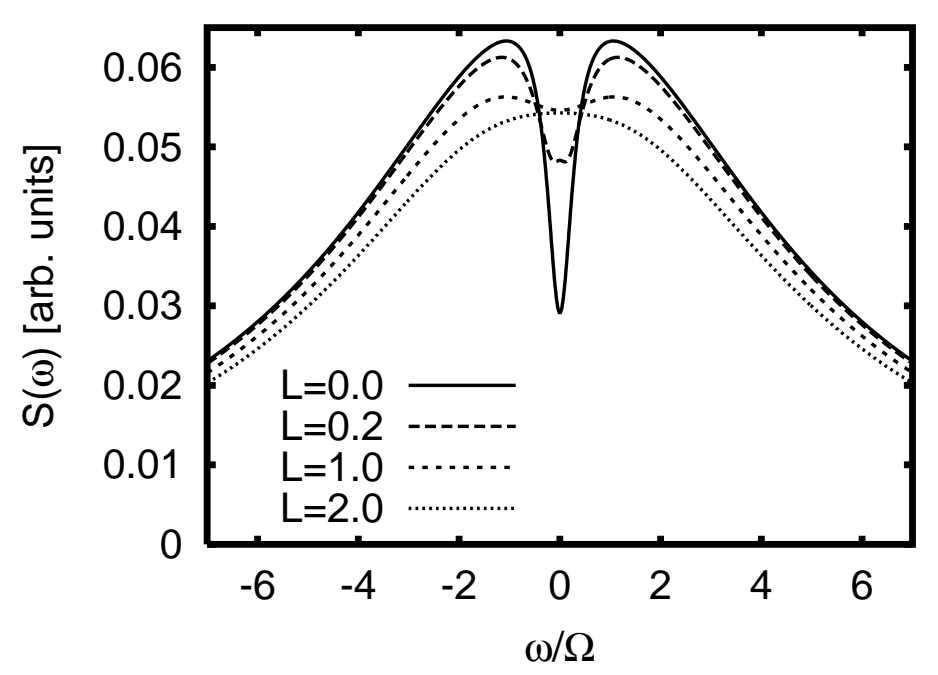

Figure 1. Resonance fluorescence spectra at parameters $\Gamma / \Omega=5, \gamma / \Omega=0.05$, $\Delta / \Omega=0$, and at various $L$ parameters: $0,0.2 \Omega, 1.0 \Omega, 2.0 \Omega$. It can be seen that, though the narrow dip in the spectra vanishes as the phase noise increases, it is relatively robust against phase noise.

of revealing time correlations between the states of the atom involved in the interfering transition channels, which are the prerequisites of quantum interference. Quantumtrajectory methods are based on the simulation of quantum trajectories, which are individual realizations of the evolution of the system conditioned on particular sequence of observed events. By tracking the time evolution of a single quantum-trajectory, the time correlations can be revealed.

An analysis based on quantum-trajectory method was presented for the phase noise free case in reference [20]. A similar analysis will be carried out here: the quantumtrajectory method of reference [24] will be applied for simulating the time evolution of the system in argument, and phase noise will also be taken into account.

In this system, a single quantum-trajectory evolves coherently according to the Hamiltonian

$$
\langle H\rangle_{\mathrm{coll}}=\hbar \Delta S^{z}+\frac{1}{2} \hbar \Omega\left(e^{-i \varphi(t)} S^{-}+S^{+} e^{i \varphi(t)}\right),
$$

interrupted by incoherent gedanken quantum measurements due to collisional noise events and spontaneous emission represented by the operators $C_{\Gamma}=2 \sqrt{\Gamma} S^{z}$ and $C_{\gamma}=\sqrt{\gamma} S^{-}$. The simulated master equation has the form

$$
\begin{aligned}
\dot{\rho}= & \frac{1}{i \hbar}\left[\langle H\rangle_{\text {coll }}, \rho\right]-\frac{1}{2}\left(C_{\Gamma}^{\dagger} C_{\Gamma} \rho+\rho C_{\Gamma}^{\dagger} C_{\Gamma}\right)+C_{\Gamma} \rho C_{\Gamma}^{\dagger}- \\
& -\frac{1}{2}\left(C_{\gamma}^{\dagger} C_{\gamma} \rho+\rho C_{\gamma}^{\dagger} C_{\gamma}\right)+C_{\gamma} \rho C_{\gamma}^{\dagger},
\end{aligned}
$$

that is equivalent to the master equation (8). The evolution of the density operator of the system is obtained by averaging the density operators of the individual quantum trajectories. The resulting density operator is the solution of the master equation (8). 
The accuracy of the simulation is limited by two factors: the length $\Delta t$ of the time step and the number $N$ of the simulated quantum trajectories. $\Delta t$ should be much less than the characteristic time of any process in the system. $N$ should be large enough to obtain the right ensemble averages for the density operator at the given stochastic noise magnitude. In our simulations $N$ was approximately $5 \cdot 10^{5}$.

In order to verify the validity of our calculations, we have also derived the spectra from the numerical results. The results obtained were equal to those obtainable analytically.

For the further considerations, it is convenient to introduce the dressed-state basis in which the Hamiltonian in (6) is diagonal in the absence of noise:

$$
\begin{aligned}
& |1\rangle=\cos \Theta|g\rangle+\sin \Theta|e\rangle \\
& |2\rangle=-\sin \Theta|g\rangle+\cos \Theta|e\rangle
\end{aligned}
$$

where

$$
\Theta=-\frac{1}{2} \arctan \left(\frac{\Omega}{\Delta}\right)
$$

An atomic state can be expanded as

$$
|\psi\rangle=c_{1} e^{i \varphi_{1}}|1\rangle+c_{2} e^{i \varphi_{2}}|2\rangle
$$

in this basis, where $c_{1}, c_{2}, \varphi_{1}, \varphi_{2}$ are real numbers. The phase difference and phase sum for the particular state may then be defined as

$$
\begin{aligned}
& \Delta \varphi=\varphi_{1}-\varphi_{2}, \\
& \Sigma \varphi=\varphi_{1}+\varphi_{2} .
\end{aligned}
$$

The phase difference can be calculated straightforwardly from a single quantumtrajectory. It was shown in reference [20, that in the lack of phase noise, the collisional noise can unexpectedly create phase correlation between the neighboring atomic dressed states. This is the underlying physical process that makes the quantum interference possible, which leads to a narrow dip in the spectrum. It is then natural to ask whether phase noise destroys the phase correlation.

The behavior of the phase difference (32) for different settings of the noise parameters are shown in figures 215. One can observe, that in the lack of both noise processes (figure 21) the distribution of phase difference shows no structure: the time evolution of the phase difference is linear due to Rabi oscillations, interrupted only by decays to the ground state due to spontaneous emission. In the case of dominant collisional noise (figure [3), the phase difference tends to stabilize around the values 0 and $\pi$. Note the symmetry of the graph originating from the phase flip caused by the operator $S^{z}$ that corresponds to collisional events. The operator $S^{z}$ exchanges the two dressed states at resonance $(\Delta=0): 2 S^{z}|1\rangle=|2\rangle$ and vica versa. In the high phase noise without collisional noise (figure 4), the phase difference tends to stabilize around the value $\pi$ only. The most interesting case from our point of view is, however, the fourth case (figure 5), when both noises are relevant. The structure of the phase distribution is 
apparently similar to that without the phase noise. From this we can conclude, that the phase correlation created by collisions is indeed not completely destroyed by the phase noise.

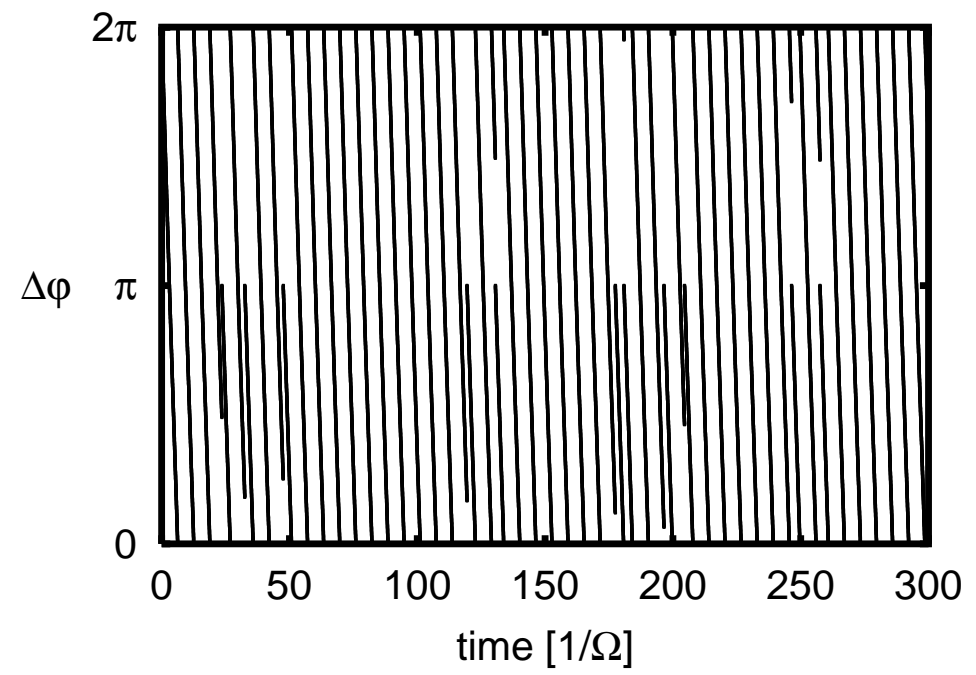

Figure 2. The phase difference $\Delta \varphi$ between the dressed states for one quantumtrajectory in the case of no phase noise and no collisional noise $(L / \Omega=0, \Gamma / \Omega=0$, $\Delta / \Omega=0, \gamma / \Omega=0.05)$.

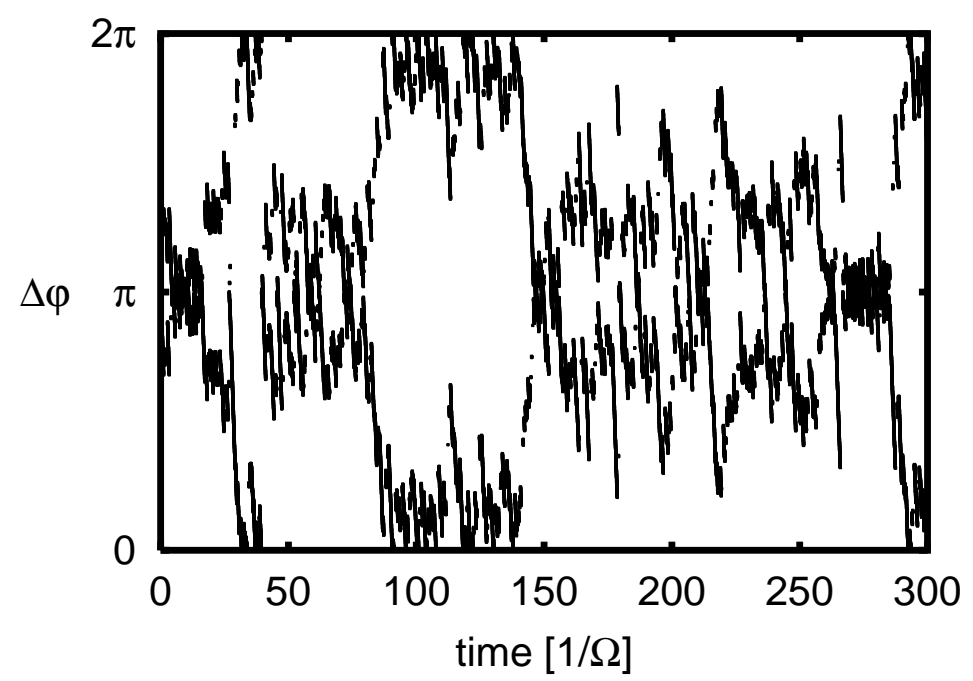

Figure 3. The phase difference $\Delta \varphi$ between the dressed states for one quantumtrajectory in the case of dominating collisional noise $(L / \Omega=0, \Gamma / \Omega=5, \Delta / \Omega=0$, $\gamma / \Omega=0.05)$.

Similarly as in Ref. [19], the phase stabilisation around the 0 and $\pi$ phase differences can be characterized explicitly by the time-correlation function of the cosine of the phase 


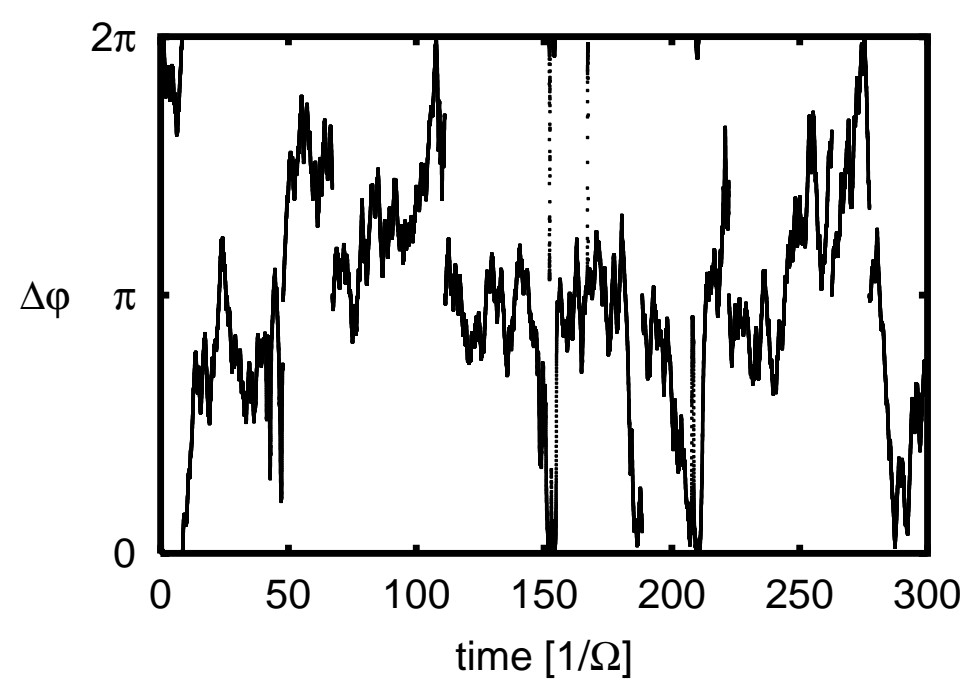

Figure 4. The phase difference $\Delta \varphi$ between the dressed states for one quantumtrajectory in the case of dominating phase noise $(L / \Omega=5, \Gamma / \Omega=0, \Delta / \Omega=0$, $\gamma / \Omega=0.05)$.

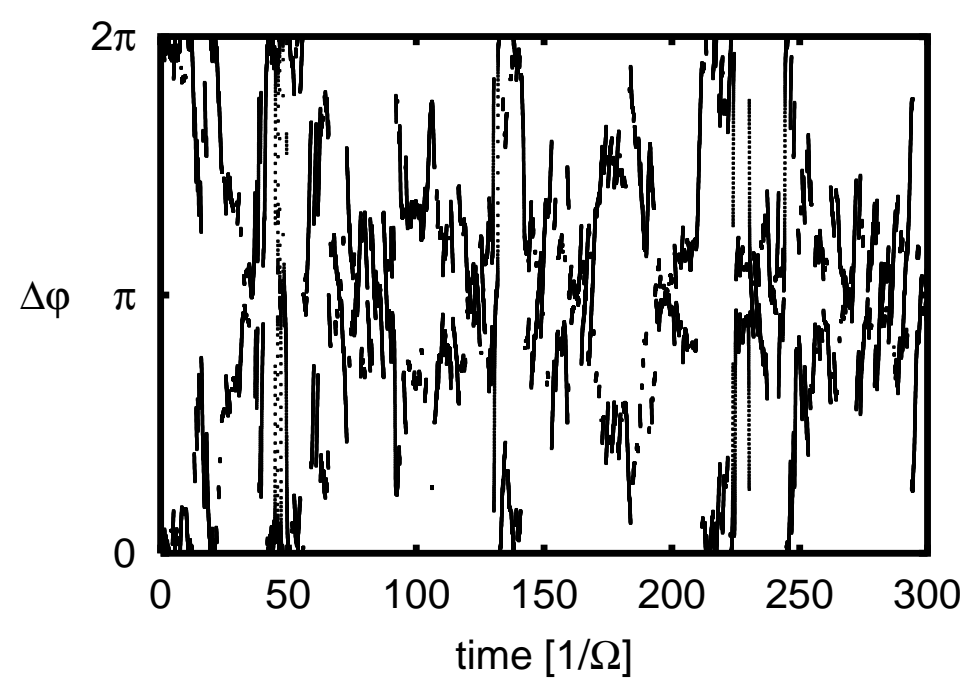

Figure 5. The phase difference $\Delta \varphi$ between the dressed states for one quantumtrajectory in the case of simultaneous phase noise and collisional noise $(L / \Omega=0.5$, $\Gamma / \Omega=5, \Delta / \Omega=0, \gamma / \Omega=0.05)$.

difference, defined as

$$
\begin{aligned}
C_{\cos }(\tau)= & c \int_{t=0}^{T}(\cos \Delta \varphi(t+\tau)-\overline{\cos \Delta \varphi}) \\
& \times(\cos \Delta \varphi(t)-\overline{\cos \Delta \varphi}) d t
\end{aligned}
$$

depicted in figure 6. The figure shows that the phase noise also leads to phase correlation between the dressed states, moreover, the phase stabilisation persist even when both collisional and phase noises are present in the system. This on the other hand gives rise to another question, namely, what is the real reason of the disappearance of quantum 
interference. In order to answer this question, let us transform the Hamiltonian (6) at

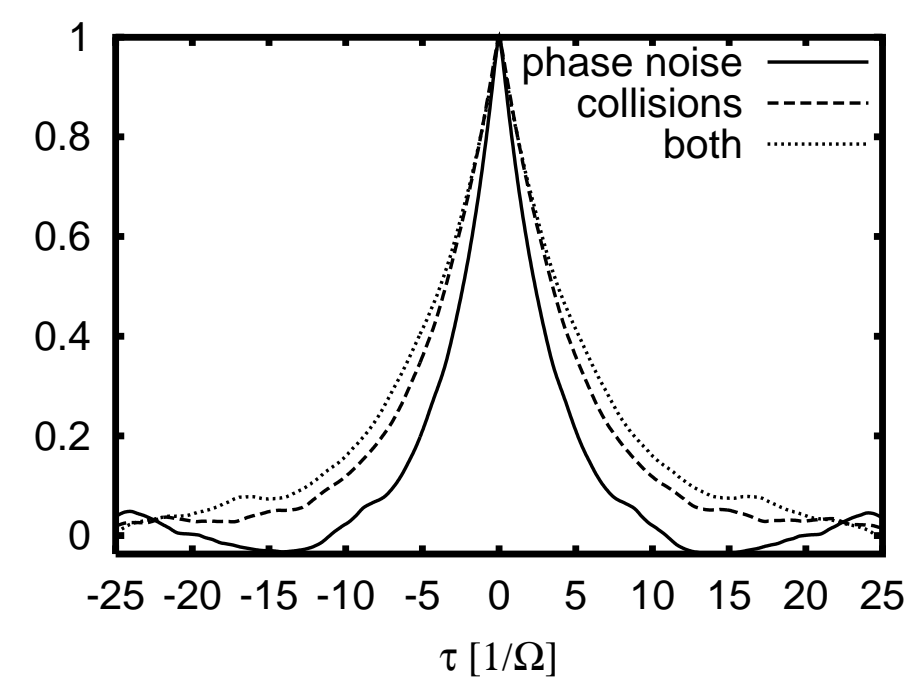

Figure 6. The time-correlation function of the cosine of the phase difference is shown in the following cases: collisional noise only $(L=0, \Gamma / \Omega=5)$, phase noise only $(L / \Omega=5, \Gamma=0)$, and both noise sources turned on $(L / \Omega=5, \Gamma / \Omega=5)$. The cosine of the phase is correlated in all three cases quite equally. The remaining parameters were set to $\Delta=0$, and $\gamma / \Omega=0.05$.

resonance $(\Delta=0)$ to the dressed state basis:

$$
\begin{aligned}
H= & -\frac{1}{2} \hbar \delta \omega_{a}(t)(|1\rangle\langle 2|+| 2\rangle\langle 1|)+\frac{1}{2} \hbar \Omega((\cos \varphi(t)|1\rangle-i \sin \varphi(t)|2\rangle)\langle 1|+ \\
& (\cos \varphi(t)|2\rangle+i \sin \varphi(t)|1\rangle)\langle 2|) .
\end{aligned}
$$

It can be seen that the collisional noise $\delta \omega_{a}(t)$ swaps the dressed states without disturbing them. Phase noise generates random rotations in the dressed state basis, mixing the dressed states. This dressed state mixing can be illustrated by the phase sum of the dressed states during the time evolution of a single quantum-trajectory. The phase sum remains constant in collisions that swap the dressed states by the action of $C_{\Gamma}$. But if mixing occurs, the phase sum will not remain constant. The phase sum in case of no phase noise $(L=0)$ is depicted in figure 7 , in case of small phase noise $(L=0.2)$ it is shown in figure 8 . The jumps in the figures correspond to spontaneous emission events.

Keeping in mind the above considerations, we are now ready to explain the noiseinduced quantum interference phenomenon in the case when both collisional and phase noises are present. Resonance fluorescence of a strongly-driven two-level atom is emitted in cascade transitions downward the ladder of the dressed-state doublets. Figure 9 shows two adjacent doublets and all possible spontaneous and noise-induced transitions between the dressed-atom states. Double arrows in figure 9 represent transitions between the dressed states $|1\rangle$ and $|2\rangle$ generated by the collisional noise events. In the high noise regime, for some time intervals the phase difference tends to stabilize not only in the case when either of the noise types are present separately, but also 


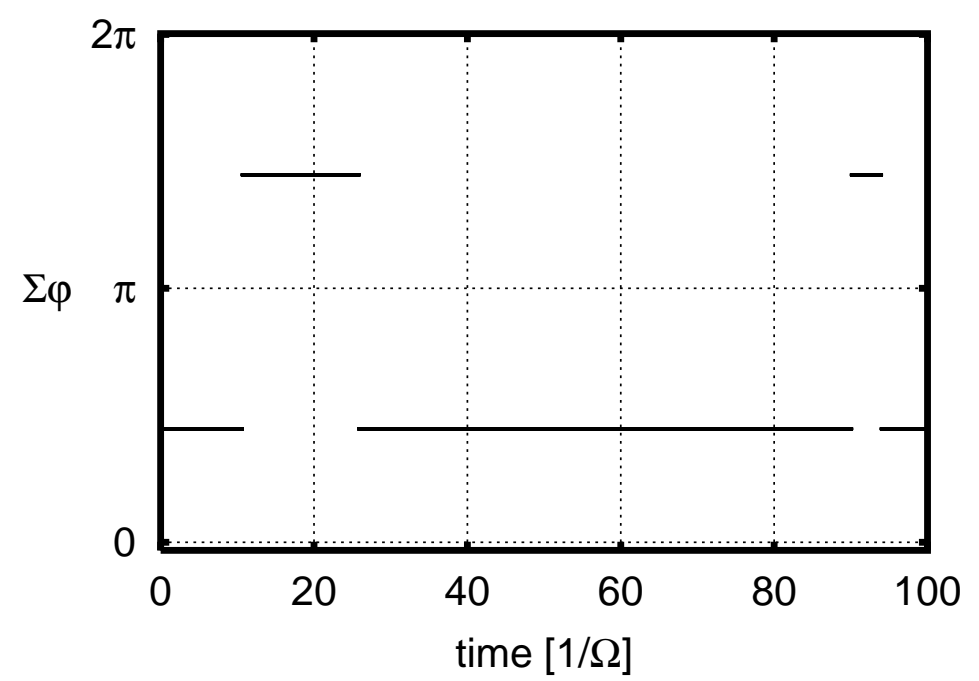

Figure 7. The sum of phases in dressed state basis for a single quantum-trajectory. There is no phase noise $(L=0)$ in the depicted case, the remaining parameters were set to $\Omega=1, \Delta=0, \Gamma=5$, and $\gamma=0.05$. The phase sum remains constant between spontaneous emissions.

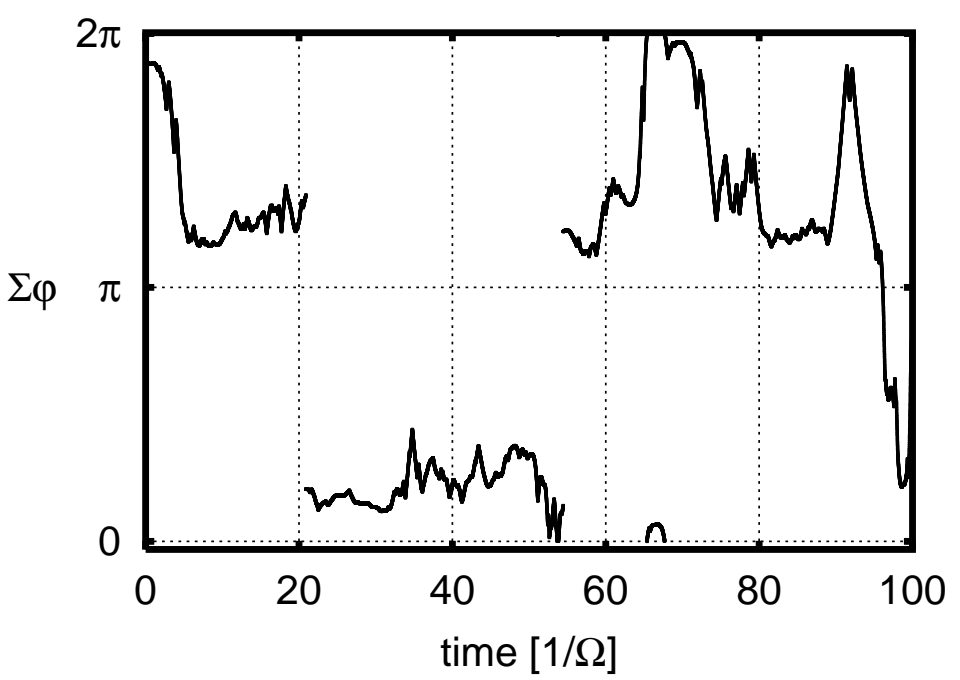

Figure 8. The sum of phases in dressed state basis for a single quantum-trajectory. There is a small phase noise $(L / \Omega=0.05)$ in the depicted case, the remaining parameters were set to $\Delta=0, \Gamma / \Omega=5$, and $\gamma / \Omega=0.05$. The phase sum changes between spontaneous emissions, showing that dressed state mixing occurs due to the phase noise. 
when simultaneously both of them are present. If phase noise is relatively low and

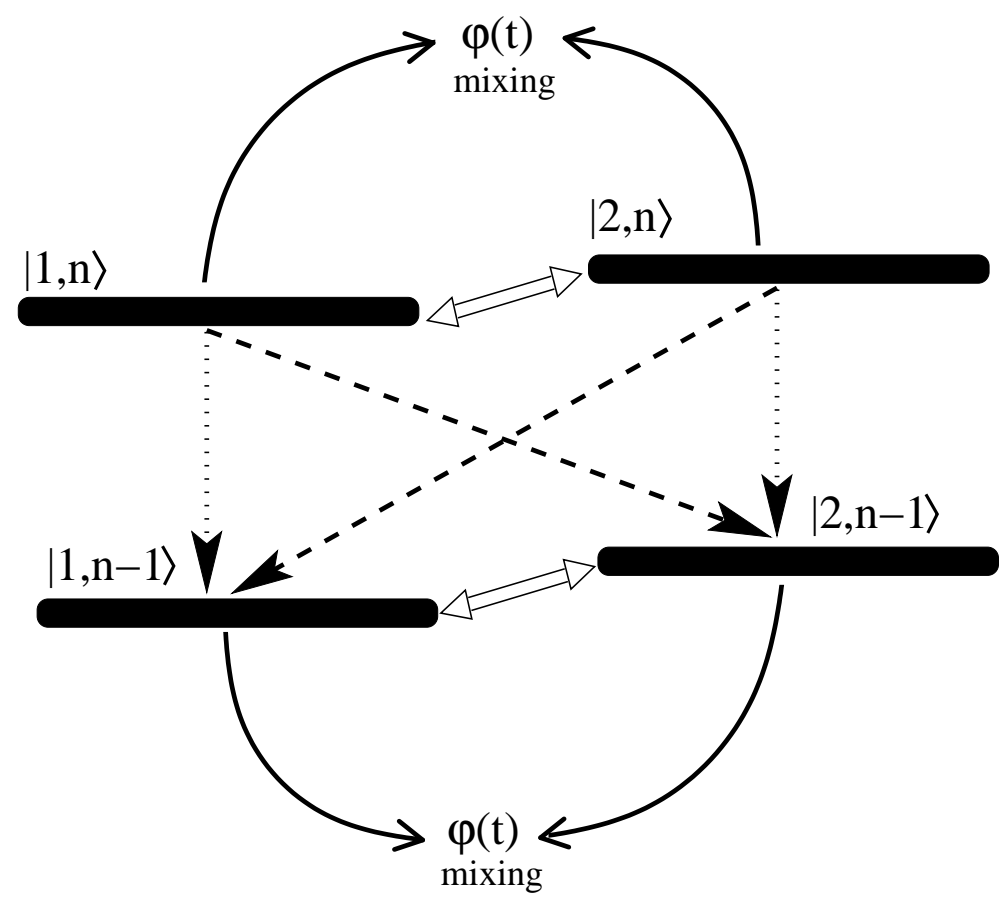

Figure 9. Different dressed-state transition channels for a coherently driven and stochastically perturbed two-level atom. The double-stroke arrows correspond to the transitions generated by collisional noise. The phase noise $\phi(t)$ mixes the dressed state doublets.

dressed-state mixing due to phase noise can be neglected, the two pairs of emission channels $|1, n\rangle \rightarrow|2, n-1\rangle \rightarrow|1, n-1\rangle$ and $|1, n\rangle \rightarrow|2, n\rangle \rightarrow|1, n-1\rangle$ (or $|2, n\rangle \rightarrow|1, n\rangle \rightarrow|2, n-1\rangle$ and $|2, n\rangle \rightarrow|1, n-1\rangle \rightarrow|2, n-1\rangle)$ differ exclusively by time ordering between collisional mixing and photon emissions. Photons emitted along these channels are indistinguishable, their destructive interference results in the dip of the fluorescence spectrum. Increasing phase noise mixes the dressed states, thus the two channels degenerate to one channel between two mixed states. Therefore quantum interference disappears. The mixing of channels, however, becomes relevant only in case of relatively high level of phase noise, thus the resonance effect is robust against phase noise.

\section{Summary}

We have analyzed the quantum interference induced by collisions in a system of coherently driven two level atoms, in the presence of phase noise in the driving field. Solving the master equation of the system in argument analytically, we have derived the resonance fluorescence spectra. There is a dip in the spectrum when the collisional noise dominates the Rabi oscillations, in the resonant case. The dip, introduced by the collisional noise, disappears relatively slowly as phase noise appears in the driving field. 
In order to understand the physical reason behind the relative robustness of the dip against the phase noise, the underlying quantum interference phenomena have been investigated. A quantum-trajectory simulation has been carried out, enabling us to investigate the phase relations between the dressed states involved in quantum interference phenomena. We have found that the phase noise does not demolish the phase correlations introduced by the collisional noise. Moreover, phase noise, similarly to the collisional noise, is of a dressed-state phase-difference stabilizing nature. On the other hand, it mixes the dressed-state doublets involved in the quantum interference, which deteriorates quantum interference in the high-noise limit.

\section{Acknowledgements}

This work was supported by the Research Fund of Hungary under contract No. T034484 and by the Polish Committee for Scientific Research (grant PBZ/KBN/043/PO3/2001). It is also a part of a general program of the National Laboratory of AMO Physics in Torun, Poland. We thank Matyas Koniorczyk for the stimulating discussions.

\section{References}

[1] Arimondo E 1996 in Progress in Optics XXXV edited by E. Wolf (Elsevier, Amsterdam, 1996) p. 257

[2] Scully M O and Zhu S Y 1998 Science 2811973

[3] Cardimona D A, Raymer M G and Stroud C R 1982 J. Phys. B-At. Mol. Opt. Phys. 1555

[4] Imamoglu A 1989 Phys. Rev. A 402835

[5] Scully M O, Zhu S Y and Gavrielides A 1989 Phys. Rev. Lett. 622813

[6] Harris S E 1989 Phys. Rev. Lett. 621033

[7] Mandel P and Kocharovskaya O 1993 Phys. Rev. A 475003

[8] Toor A H, Zhu S Y and Zubairy M S 1995 Phys. Rev. A 524803

[9] Zhou P and Swain S 1997 Phys. Rev. Lett. 78832

[10] Zhu S Y, Chan R C F and Lee C P 1995 Phys. Rev. A 52710

[11] Zhu S Y, Narducci L M and Scully M O 1995 Phys. Rev. A 524791

[12] Xia H R, Ye C Y and Zhu S Y 1996 Phys. Rev. Lett. 771032

[13] Zhu S Y and Scully M O 1996 Phys. Rev. Lett. 76388

[14] Zhou P and Swain S 1996 Phys. Rev. Lett. 773995

[15] Zhou P and Swain S 1997 Phys. Rev. A 563011

[16] Alzetta G, Gozzini, Moi L, and Orriols G 1976 Nuovo Cimento 36B 5

[17] Prior Y, Bogdan A R, Dagenais M and Bloembergen N 1981 Phys. Rev. Lett. 46111

[18] Wilson-Gordon A D and Friedmann H 1983 Opt. Lett. 8617

[19] Grynberg G in Spectral Line Shapes edited by Exton R (W. De Gruyter, Berlin, 1987), Vol. 4, p. 503

[20] Karpati A, Adam P, Gawlik W, Lobodzinski B and Janszky J 2002 Phys. Rev. A 66023821

[21] Carmichael H J An Open System Approach to Quantum Optics Vol. M18 of Springer Lecture Notes in Physics (Springer-Verlag, Berlin, 1993)

[22] Plenio M B and Knight P L 1998 Rev. Mod. Phys. 70101

[23] Garraway B M and Knight P L 1994 Phys. Rev. A 491266

[24] Dalibard J, Castin Y and Mølmer K 1992 Phys. Rev. Lett. 68580

[25] Gardiner C W, Parkins A S and Zoller P 1992 Phys. Rev. A 464363

[26] Dum R, Zoller P and Ritsch H 1992 Phys. Rev. A 454879 
[27] Gawlik W, Lobodzinski B and Chałupczak W in Frontiers of Quantum Optics and Laser Physics edited by Zhu S, Scully M and Zubairy M (Springer, Berlin, 1997)

[28] Elliott D S and Smith S J 1988 J.O.S.Am.B 51927

[29] Agarwal G S 1976 Phys. Rev. Lett. 371383

[30] Eberly J H 1976 Phys. Rev. Lett. 371387

[31] Kimble H J and Mandel L 1977 Phys. Rev. A 15689

[32] Knight P L, Molander W A and Stroud C R 1978 Phys. Rev. A 171547

[33] Toor A H and Zubairy M S 1994 Phys. Rev. A 49449

[34] Zhou P, Fang M F, Zhou Q P and Li G X 1999 Phys. Lett. A 251199

[35] Agarwal G S 1978 Phys. Rev. A 181490

[36] Mollow B R 1969 Phys. Rev. 1881969 